\title{
ADAPTIVE SUPPRESSION OF CCD SIGNAL-DEPENDENT NOISE IN LIGHT SPACE
}

\author{
Hilda Faraji and James MacLean \\ Department of Electrical and Computer Engineering, \\ University of Toronto, 10 Kings College Road, Toronto, ON, M5S 3G4
}

\begin{abstract}
We propose a denoising scheme to restore images degraded by CCD noise. Typically, restoration algorithms assume a linear mapping between the incident light space and image space. However, in practice a camera response function performs a non-linear mapping on the sensor output and as a result the sensor noise model becomes more complex in the image space. In this paper, we correct for non-linearity by mapping the corrupted image into "light space", where the relationship between the incident light and light space values is linear. To reduce the sensor noise we accurately model the CCD sensor noise by using the Photon Transfer Curve. We then develop a combination of adaptive filters based on the estimated noise model in light space. Our adaptive system demonstrates efficient noise removal performance in uniform regions, while preserving edges and fine details.
\end{abstract}

\section{INTRODUCTION}

Digital images are prone to a variety of CCD noise sources, which can seriously restrict the ability to achieve high quality images with commercial CCDs [1]. A general model for a CCD camera system typically includes a sensor, a nonlinear response function, and noise components as shown in Fig. 1. Light photons strike the CCD sensor and generate sensor data $q$, which represents the quantity of light integrated over the spectral response of the camera. The sensor data, $q$, are subjected to CCD sensor noise sources such as the random arrival of photons, dark current noise, amplifier noise, and Fixed Pattern Noise (FPN) [1]. The noisy data then pass through the nonlinear camera response function $\mathcal{F}$ to form the image. This image is then converted to the final digital image $I$ by an A/D conversion unit. This conversion introduces quantization noise, but this is negligible in comparison with sensor noise [2]. Denoising images degraded by CCD noise requires an appropriate sensor noise model. To obtain this model, access to the sensor data is necessary. Most restoration algorithms assume that the image value or brightness $I$ is a linear function of $q$ [3]. In this case the noisy image values are proportional to the sensor data and the strategy for the estimation of sensor data can be implemented in the image space. In contrast, if the linear

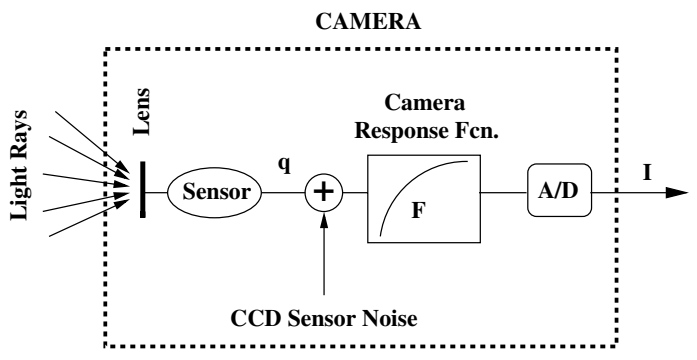

Fig. 1. Nonlinear camera response function causes image values to be nonlinearly dependent on sensor data.

assumption is violated, the image noise model will be different from the sensor noise model and can be somewhat more complex. This motivates the estimation of the sensor data $\hat{q}$ by applying the inverse of the nonlinear camera response function, $\mathcal{F}$, to the image data $(I)$, so that $\hat{q}=\mathcal{F}^{-1}(I)$. The converted image values, $q$, lie in a new space called the "light space" [2]. For applications where the sensor data is required or where defect in the image originates in light space, processing the raw camera responses leads to more satisfactory results [2], and [4].

\section{ADAPTIVE FILTERING IN LIGHT SPACE}

We use adaptive filters to learn the local signal and noise characteristics so that the filter parameters can be adjusted accordingly. Our denoising algorithm consists of three main stages as shown in Fig. 2. A noisy image is initially converted to light space, then processed using a combination of Local Linear Minimum Square Error (LLMSE) filters with different window sizes and an adaptive Wiener filter. The final image is a weighted average of individual output images through a procedure explained in Section 2.3. The following sections will describe these procedures in detail.

\subsection{Estimation of CCD sensor noise model}

We build the sensor noise model using a Photon Transfer Curve (PHTC) [1]. This curve is a plot of noise standarddeviation against the noise-free sensor data, $q$. An example of a PHTC for a Kodak DCS260 is shown in Fig. 3. A PHTC typically includes three parts, depending on the strength of 


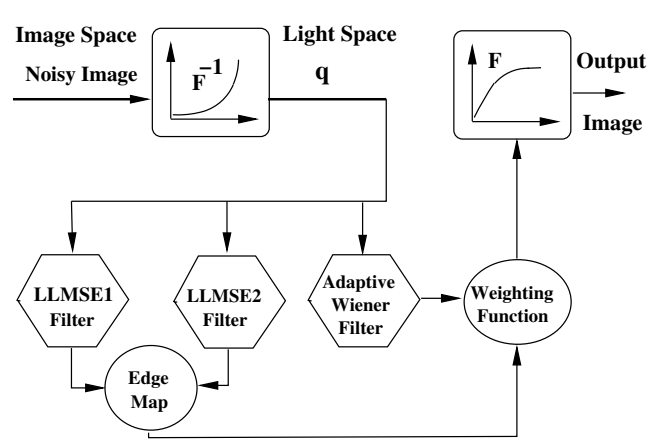

Fig. 2. Our general filtering scheme in light space.

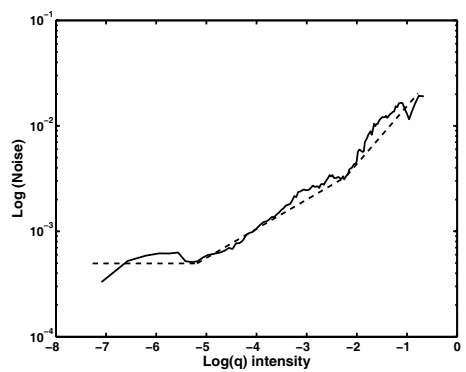

Fig. 3. Photon transfer curve (Kodak DCS260) in the logarithmic scale. Dotted lines denote the linear approximations to the three regions. The $q$ values are normalized to the range $[0,1]$

the signal [1]. For low signal levels, the curve is almost flat, implying that noise (mostly amplifier noise) in the dark areas of an image is independent of the $q$ values. As the illumination increases, noise becomes more signal dependent (nonzero slope). Shot noise is dominant in this middle region. The third part of the curve has a sharp rise in comparison with previous regions. Fixed Pattern Noise (FPN) is the dominant noise in this regime.

Since FPN can be removed by a pre-processing procedure [1], we can reasonably assume a two-region PHTC in which the third region has the same noise characteristics as the second one. A set of images of a standard uniform object with different exposure times are used to generate the PHTC. Every image is converted to light space. Under the assumption of zero-mean noise, the noise-free sensor data are then estimated as the average of $q$ values in each light space image. Since the scene object is uniform, the image variance can be used to model the total sensor noise variance.

From the PHTC it is evident that CCD noise variance is a function of $q$ and therefore CCD noise is signal-dependent. Most types of Signal-Dependent Noise (SDN) can be expressed in parametric form as in [5]:

$$
g(n)=f(n)+\eta(n)
$$

$$
\eta(n)=\sigma_{u} \cdot f^{\gamma}(n) \mathcal{N}(n)
$$

where the index $n$ denotes the image coordinate, $g$ refers to the noisy image, and $f$ is the unknown noise free image. Sensor noise $\eta$, is defined as a function of $f$ and a zero-mean unity variance Gaussian noise $\mathcal{N}$. Noise parameters $\sigma_{u}, \gamma$, and $\sigma_{c}$ are determined using the PHTC. For instance, on the logarithmic scale, the intercept of PHTC is $\sigma_{u}$ and $\gamma$ is the slope in the middle region. Let $\sigma_{c}$ denote the noise standarddeviation in the first region of the PHTC, ignoring FPN, the noise standard deviation $\sigma_{n}$ associated with a typical CCD sensor can be formulated as

$$
\sigma_{n}=\left\{\begin{array}{ll}
\sigma_{c} & f \leq e^{B} \\
\sigma_{u} \cdot f^{\gamma} & f>e^{B}
\end{array} .\right.
$$

When the image intensity is less than a certain value $e^{B}$, noise can be modeled as constant.

\subsection{Filtering approach}

Based on a typical CCD noise model, we describe two filtering approaches to address each noise region. The first approach deals with SDN, while the latter removes the constant noise from the degraded image in the first noise regime.

Many attempts have been made to restore the images degraded by SDN [6], [7], and [8]. In [8], a LLMSE filter is applied in the wavelet domain to restore images degraded by SDN whose model is denoted in Eqn. 2. Uniform patches are determined from the noisy observation, and used to estimate the noise parameters $\gamma$ and $\sigma_{u}$ [9]. However detecting the uniform image regions from the noisy image is challenging when dealing with SDN. Consequently the estimated noise parameters may not be accurate. In this work we accurately estimate the noise parameters using the PHTC as mentioned in Section 2.1. We also apply a spatial adaptive filter in light space using the same filter structure as explained in [8]. In particular, the filter formulation is

$$
\hat{f}_{L L M S E}(n)=\left(1-\frac{\sigma_{f}^{2}(n)}{\sigma_{g}^{2}(n)}\right) \mu_{f}+\frac{\sigma_{f}^{2}(n)}{\sigma_{g}^{2}(n)} \cdot g(n)
$$

where $n$ refers to the image index, $\hat{f}_{L L M S E}$ is the noise-less estimate, $g$ is the noisy image, $\sigma_{f}^{2}$ and $\mu_{f}$ are the noisefree local statistics, which need to be estimated based on the noisy local variance $\sigma_{g}^{2}$, local mean $\mu_{g}$, and prior knowledge of noise model. Since we assume that the Gaussian noise is independent of the noise-free image $f$, there is no correlation between $f$ and the Gaussian noise, thus $\mu_{f}=\mu_{g}$. Furthermore $\sigma_{g}^{2}(n)$ can be written as

$$
\sigma_{g}^{2}=\sigma_{f}^{2}+\sigma_{u}^{2} \mathrm{E}\left[f^{2 \gamma}\right] .
$$

We drop $n$ for notational simplicity. To simplify the expression for $\mathrm{E}\left[f^{2 \gamma}(n)\right]$, we use the Taylor series expansion of $f^{2 \gamma}$ around $\mu_{f}$, so

$$
E\left[f^{2 \gamma}\right] \approx \mu_{f}^{2 \gamma}+\gamma(2 \gamma-1) \mu_{f}^{2 \gamma-2} \sigma_{f}^{2} .
$$


Finally $\sigma_{g}^{2}$ can be summarized as

$$
\begin{aligned}
\sigma_{g}^{2} & =\sigma_{f}^{2}(n)+\sigma_{u}^{2} \mathrm{E}\left[f^{2 \gamma}(n)\right] \\
& =\sigma_{f}^{2}+\sigma_{u}^{2}\left(\mu_{f}^{2}+\gamma(2 \gamma-1) \mu_{f}^{2 \gamma-2} \sigma_{f}^{2}\right) \\
\rightarrow \sigma_{f}^{2} & =\frac{\sigma_{g}^{2}-\sigma_{u}^{2} \mu_{f}^{2 \gamma}}{1+\gamma(2 \gamma-1) \mu_{f}^{2 \gamma-2} \sigma_{u}^{2}} .
\end{aligned}
$$

Therefore $\hat{f}_{L L M S E}$ is a function of $\sigma_{g}^{2}, \mu_{g}$, and the noise parameters:

$$
\hat{f}_{L L M S E}(n)=\frac{\left(1-\sigma_{u}^{2} \cdot \frac{\mu_{g}^{2 \gamma}}{\sigma_{g}^{2}}\right)\left(g(n)-\mu_{g}\right)}{1+\gamma(2 \gamma-1) \mu_{g}^{2 \gamma-2} \sigma_{u}^{2}}+\mu_{g} .
$$

As indicated by Eqn 4, the filter adapts to local statistical variations such that uniform regions are smoothed, while edges and fine details are invariant to the averaging process. When the local image region is uniform, the estimated $\sigma_{f}^{2}$ is considerably smaller than the corresponding $\sigma_{g}^{2}$, causing the ratio $\frac{\sigma_{f}^{2}}{\sigma_{g}^{2}}$ to be close to zero. Thus the filter puts most of the weight on $\mu_{g}$, smoothing the areas. Conversely, in the presence of a sharp edge, $\frac{\sigma_{f}^{2}}{\sigma_{g}^{2}}$ is close to one, and as a result the filter puts more weight on the noisy values, $g$. In this case the edge sharpness is preserved.

However, LLMSE filters based on Eqn. 8 perform poorly in dark areas, because noise in dark areas is signal-independent. We apply a standard adaptive Wiener filter to deal with signalindependent noise with known variance $\sigma_{c}$. This filter creates a pixel-wise Wiener filter using the local estimates $\mu_{g}$ and $\sigma_{g}^{2}[5]$ :

$$
\hat{f}_{\text {Wiener }}(n)=\mu_{g}+\frac{\sigma_{g}^{2}(n)-\sigma_{c}^{2}}{\sigma_{g}^{2}(n)}\left(g(n)-\mu_{g}(n)\right) .
$$

\subsection{Merging maps}

In addition to the filter structure, the window size also affects the filter performance. As mentioned earlier, the LLMSE filter prevents edge blurriness by assigning less weight to $\mu_{g}$ in non-uniform regions, however this creates artifacts in the vicinity of sharp edges. The distortion width around the edges depends on the window size of the filter. The bigger the window size, the broader the distorted region. Employing an LLMSE filter with small window size would reduce this problem at the expense of poor averaging performance due to the reduced number of samples in each local patch. To perform well in uniform regions, while reducing the artifacts, we apply two LLMSE filters with $3 \times 3$ and $9 \times 9$ window sizes. We then provide a smooth edge map to blend the outputs of these two filters. The results are combined so that the filter with smaller window size is dominant in edge regions, but gradually reduces its influence in uniform regions where the second filter becomes dominant. To do this, a binary edge map is initially generated by thresholding the ratio between the estimated $\sigma_{f}^{2}$ to $\sigma_{g}^{2}$. This binary map is then morphologically dilated [10] to let the edges diffuse throughout a small neighborhood, creating a smooth transition between the two LLMSE filters as shown in Fig. 4. The output image at this stage is a local nonlinear MSE filter, LNMSE.

The final LNMSE filter result is then merged with the results of the adaptive Wiener filter result (Fig. 2). A sigmoidlike weighting function assigns all the weight to the Wiener filter where the signal-independent noise is the dominant noise. For image intensities close to $e^{B}$, the output is the weighted average of two filters. The LNMSE filter is dominant for image values beyond $e^{B}$. The weight graph is shown in Fig. 4 (right). This approach is similar to the work in [11], which is based on a more simplistic CCD noise model assumption. Edges are preserved by using a surface diffusion model combined with a min/max curvature, where the diffusion rate varies as a function of edges.
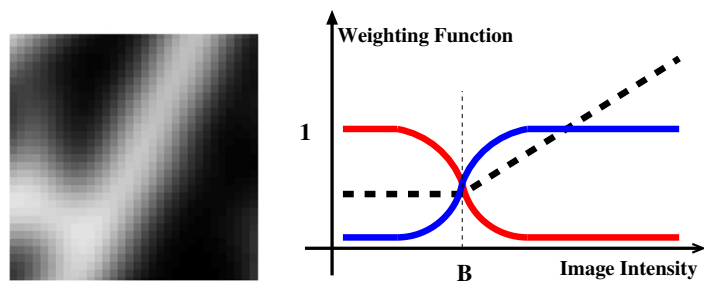

Fig. 4. (left) Cropped "Lena" edge map. (right) Sigmoid-like weighting functions (solid curves) provides a smooth mapping around the breakpoint $e^{B}$ between the adaptive Wiener and $\mathrm{LN}$ MSE filters.

\section{SIMULATION RESULTS}

For experiments, a set of noise-free images are initially converted to light space. Synthetic noise, based on the model shown in Fig. 3, is then added to the images in light space. We then process the degraded images by applying two LLMSE filters and a standard adaptive Wiener filter in light space. The results are then combined using the two merging maps. As shown in Fig. 5, the performance of the $3 \times 3$ LLMSE filter in uniform regions is poor, but the edges are preserved. In contrast the $9 \times 9$ LLMSE significantly smooths the uniform areas, but generates artifacts in edge regions. Combining the two LLMSE filters deals with the artifact problem. Fig. 6 shows how the weight function combines the results of the LNMSE, and adaptive Wiener filters. As shown, the adaptive Wiener filter blurs the image, while the LNMSE filter performs poorly in dark regions. These problems are removed by applying this map on both output images and combining the results. Overall, these figures illustrate that our final combined filter outperforms adaptive Wiener, 

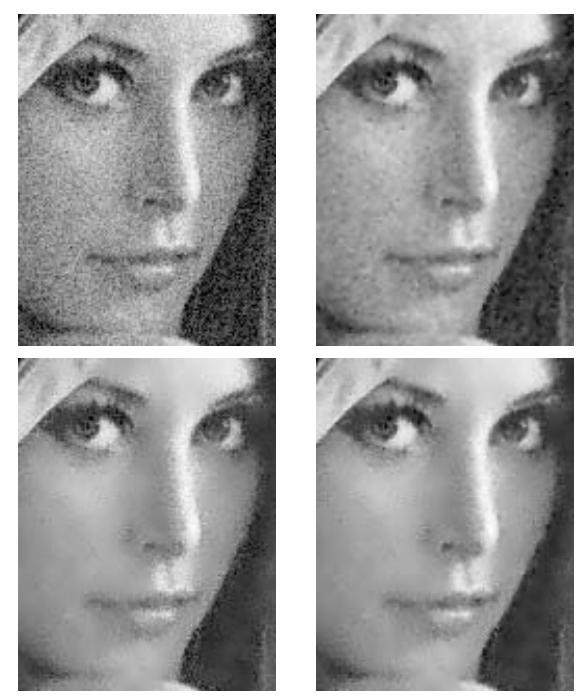

Fig. 5. Effect of edge map on artifact reduction: (top left) corrupted "Lena" image with $\gamma=0.3$ and $\sigma_{u}=0.05$, (top right) filtered image with a $3 \times 3$ LLMSE filter, (bottom left) a $9 \times 9$ LLMSE filtered image, and (bottom right) the LNMSE result.

LLMSE, and LNMSE filters by smoothing uniform regions and removing the artifacts around the edges, the blurriness effect of Wiener filter, and the remained errors in the dark regions.

We also compute Mean Square Error (MSE) as a quantitative noise-attenuation measure. Table 1 provides MSE values for various values of $\gamma$ and compares the performance of adaptive Wiener, LNMSE, and the final combined filters. As shown, noise level in the final combined image is significantly lower than the other filtered images.

Table 1. MSE measures of the "Peppers" image for three values of $\gamma$.

\begin{tabular}{ccccc}
$\gamma$ & Noisy & Wiener & LNMSE & Final combined \\
\hline 0.3 & 323.481 & 98.736 & 100.066 & 65.134 \\
\hline 0.5 & 104.889 & 94.897 & 39.488 & 38.226 \\
\hline 0.7 & 35.048 & 94.161 & 24.888 & 18.225
\end{tabular}

\section{CONCLUSION AND REMARKS}

In this paper we presented a restoration algorithm for images degraded by CCD sensor noise. Images are converted to light space, where the relationship between the incident light and the light space values is linear. Using a noise model that is based on the PHTC, light space denoising enables the design of a simpler and more straightforward restoration algorithm. Simulation results show a promising performance; uniform regions are smoothed, while abrupt changes such as edges are preserved. In future work, we plan to investigate denoising in wavelet-based light space domain.
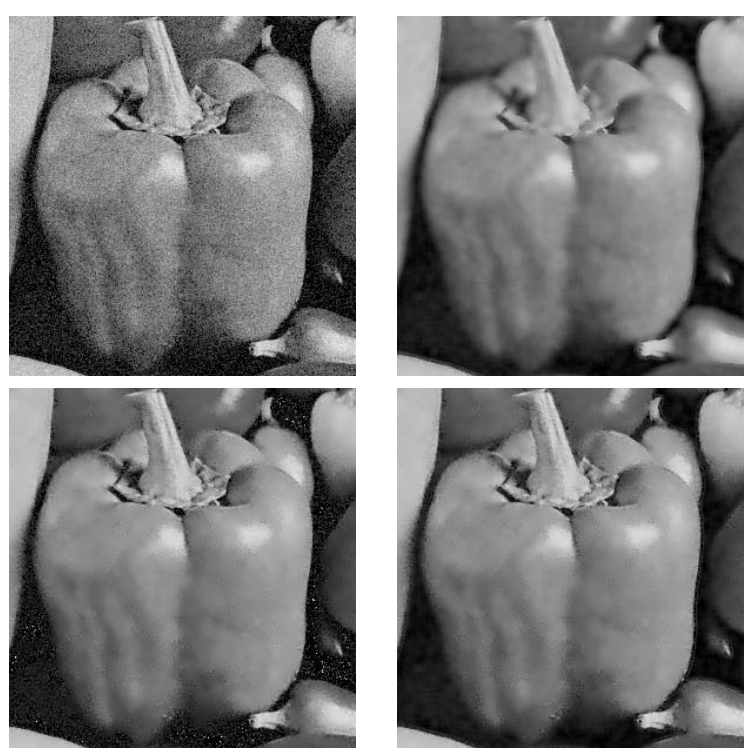

Fig. 6. Effect of the weight function: (top left) cropped "Peppers" image degraded by $\sigma_{u}=0.04$ and $\gamma=0.3$, (top right) adaptive Wiener estimate, (bottom left) LNMSE estimate, and (bottom right) final combined denoised image.

\section{REFERENCES}

[1] James R. Janesick, Scientific Charge-Coupled Devices, SPIE Press, Bellingham, Wash., 2001.

[2] Steve Mann, Intelligent Image Processing, A John Wiley and Sons, INC., 2002.

[3] S.K. Nayar T. Mitsunaga, "Radiometric self calibration," in CVPR, June 1999, pp. -380 .

[4] C.Manders and S. Mann, "Comparametric equations and quantimetric motion analysis," in ICASSP, Apr. 2003.

[5] K. Anil Jain, Fundamentals of Digital Image Processing, Prentice Hall, 1989.

[6] I. Pitas and A.N.Venetsanopoulos, "Order statistics in digital image processing," PROC.IEEE, vol. 80, no. 12, pp. 18931921, 1992.

[7] A. Alexander T. K. Darwin, "Adaptive noise smoothing filter for images with signal-dependent noise," PAMI, vol. 7, no. 2, pp. 165-177, 1985.

[8] L. Alparone F. Argenti, G. Torricelli, "Signal-dependent noise removal in the undecimated wavelet domain," in ICASSP, May 2002, pp. IV-3293-IV-3296.

[9] S. Baronti B. Aiazzi, L. Alparone, "A robust method for parameter estimation of signal-dependent noise models in digital images," in PROC. IDSP, July 1997, pp. 601-604.

[10] I. Pitas and A.N. Venetsanopoulos, Nonlinear Digital Filters; Principles and Applications, Boston, MA: Kluwer, 1990.

[11] K. T. Park S. H. Lee, M. G. Kang, "Noise filtering based on surface diffusion model combined with min/max curvature," in PROC. IEEE ISCECCD, Dec. 1997, pp. 272-275. 